\title{
ESTRONGILOIDÍASE DISSEMINADA EM PACIENTE COM INFECÇÃO POR HTLV-1: RELATO DE CASO
}

\section{STRONGIUOIDIASIS DISSEMINATED INAPATIENTWITH HTLV-1 INFECTION: CASE REPORT}

\begin{abstract}
Autores
Rayane Cesário Pereira' Carolina Missio Rodriges ${ }^{1}$ Léo da Croce Neto ${ }^{1}$ Keily Stefanni Ordones Nery Mendes ${ }^{1}$ Renata Andrade Mello ${ }^{2}$
\end{abstract}

1 Residente de Clínica Médica do Hospital Santa Rosa

2 Clínica, preceptora do programa de Residência de Clínica Médica do Hospital Santa Rosa

\section{RESUMO}

A estrongiloidíase é uma helmintíase causada pelo Strongyloides stercoralis frequentemente assintomática, porém pode manifestarse como síndrome de hiperinfecção associada a acometimento de múltiplos órgãos e sistemas. Estima-se que a forma disseminada ocorra em 1,5 a 2,5\% dos pacientes, especialmente em estado de imunossupressão. Neste trabalho é descrito um caso de estrongiloidíase disseminada que se desenvolveu em paciente com infecção pelo HLTV1 (Vírus Linfotrópico da Célula T Humana), com outros fatores de risco para a doença.

Palavras-Chave: Estrongiloidíase; Imunossupressão; Infecções por HTLV-1.

\section{ABSTRACT}

Strongyloidiasis is a helmitiasis caused by Strongyloides stercoralis, which is often asymptomatic, but it can manifest itself as a hyperinfection syndrome associated with the involvement of multiple organs and systems. It is estimated that the disseminated form occurs in 1.5 to $2.5 \%$ of patients with strongyloidiasis, especially in the state of immunosuppression. This work described a case of disseminated strongyloidiasis in a patient with HLTV-1 infection, with other risk factors for the disease.

Keywords: Strongyloidiasis; Immunosuppression; HTLV-1 Infections.

\section{INTRODUÇÃO}

A estrongiloidíase é uma doença parasitária intestinal, 
1. Melo MCB, Klem VGQ, Mota JAC, Penna FJ. Parasitoses Intestinais. Rev Med Minas Gerais. 2004;14(Suppl 1):3-12.

2. Vadlamudi RS, Chi DS, Krishnaswamy G. Intestinal strongyloidiasis and hyperinfection syndrome. Clin Mol Allergy. 2006:4:8. Available from: https://pubmed.ncbi.nlm.nih gov/16734908/.

3. Brasil. Ministério da Saúde. Secretaria de Vigilância em Saúde. Departamento de Vigilância Epidemiológica. Doenças infecciosas e parasitárias: guia de bolso. 8th ed. Brasília: Distrito Federal; 2010. p.176-178. Available from: http://bvsms.saude.gov. br/bvs/publicacoes/doencas_infecciosas_ parasitaria_guia_bolso.pdf frequentemente assintomática, causada pelo Strongyloides stercoralis. As formas de transmissão são: heteroinfecção na qual as larvas presentes no solo penetram no homem pela pele, além de autoinfecção interna ou externa ${ }^{1}$.

A síndrome de hiperinfecção está relacionada a um aumento no número de larvas, associada a manifestações confinadas aos sistemas respiratório e gastrointestinal. Estima-se que ocorra em 1,5 a 2,5\% dos pacientes com estrongiloidíase, pela enorme multiplicação e migração de larvas infectantes, especialmente em um estado imunossuprimidos². Os quadros se caracterizam por febre, dor abdominal, anorexia, náuseas, vômitos, diarreias profusas e manifestações pulmonares ${ }^{3}$.

Imunossupressão por uso de corticosteroides sistêmicos ou devido a doenças como HTLV-1 e a infecção pelo HIV (Vírus da Imunodeficiência Humana), o transplante de órgãos e outras doenças infecciosas podem aumentar o risco de síndrome de hiperinfecção².

\section{RELATO DE CASO}

OJSB, masculino, 38 anos, casado, administrador, natural de Cuiabá-MT, residente em zona urbana. Admitido, em leito de enfermaria de um hospital particular, com quadro de náuseas e vômitos persistentes, epigastralgia, astenia e palidez cutânea que iniciaram há 2 semanas, associado a constipação nesse período, perda de peso não quantificada e 1 episódio de febre não termometrada. Relatava hipertensão prévia e realização de colangiopancreatografia retrógrada endoscópica (CPRE) há 7 anos por estenose ductal. De história familiar, a mãe faleceu por complicações do câncer de pulmão. $\mathrm{Na}$ história social afirmava frequentar, em todos os finais de semana, o sítio do irmão sem saneamento básico, na qual andava descalço a maior parte do tempo, além do etilismo (média de $192 \mathrm{~g}$ de álcool aos sábados e domingos).

Ao exame físico apresentou-se em regular estado geral, apático, hipocorado, desidratado, emagrecido, eupneico, anictérico, afebril, sem alterações na ausculta pulmonar e cardíaca. Os exames da admissão demonstravam anemia microcítica e normocrômica (Hb 12,1g/dL), eosinofilia (961/mm2) e hiponatremia hipotônica hipovolêmica ( $\mathrm{Na}$ $125,0 \mathrm{meq} / \mathrm{L})$. Paciente não tolerava a alimentação via oral por êmese e 
dor epigástrica associada, mesmo com medicações para proteção gástrica e antieméticos otimizados. Realizada endoscopia digestiva alta que observou lesão ulcerada em bulbo ocupando cerca de $90 \%$ da luz intestinal e prosseguiu-se com biópsia (Figura 1).
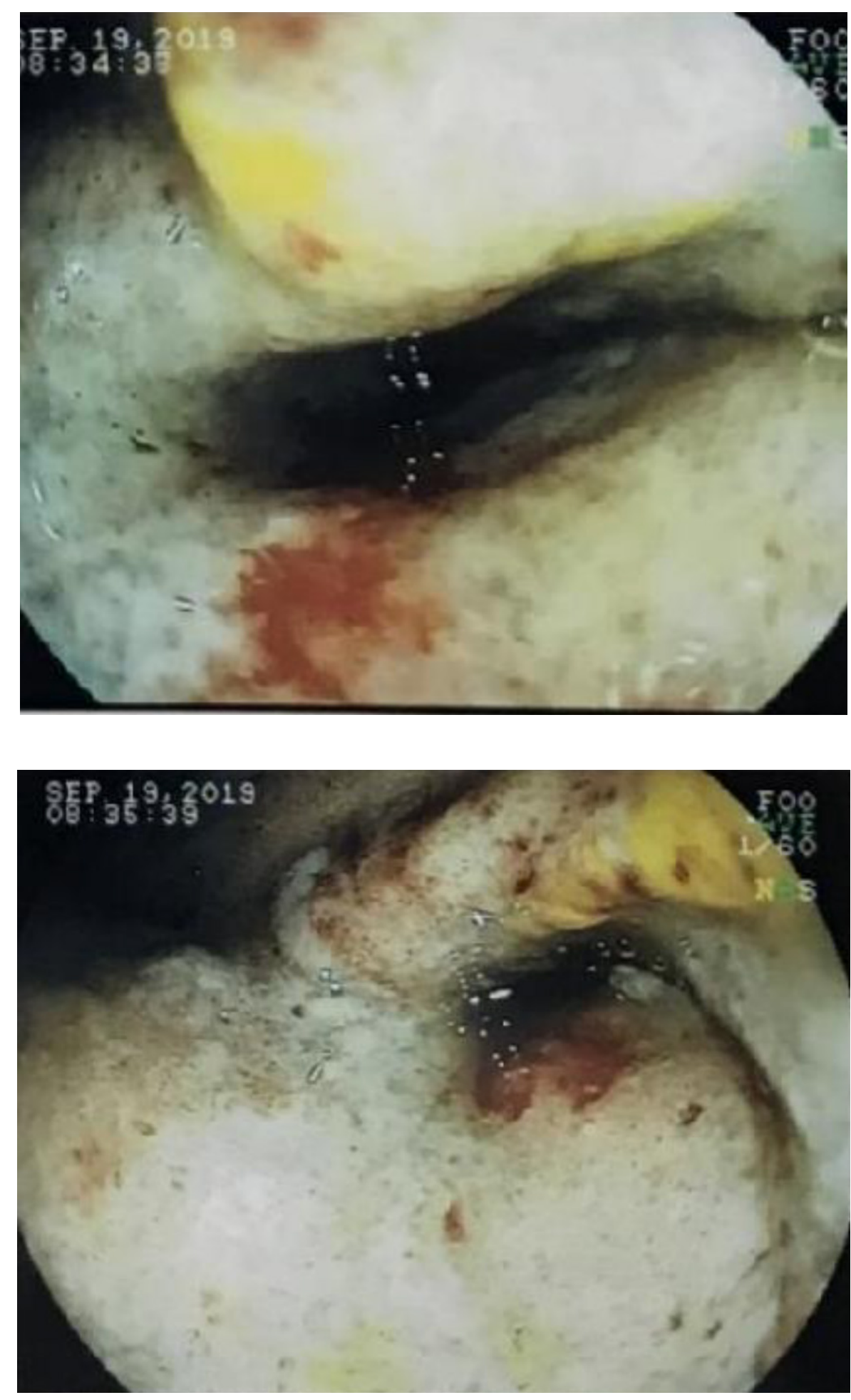

Figura 1 - Endoscopia digestiva alta: bulbo com lesão ulcerada com fibrina e edema, ocupando luz intestinal. 
Na tomografia computadorizada (TC) de abdome evidenciou aerobilia, distensão gástrica significativa, proeminência numérica de linfonodos mesentéricos e espessamento parietal de duodeno e jejuno (Figura 2). Foi iniciado tratamento com albendazol 400 mg/dia, metilprednisolona 62,5 mg/dia e mesalazina 2400 mg/dia sob hipótese de Doença de Crohn, instituída dieta parenteral por intolerância gástrica e impossibilidade de passagem de sonda nasoenteral por estenose bulbar.
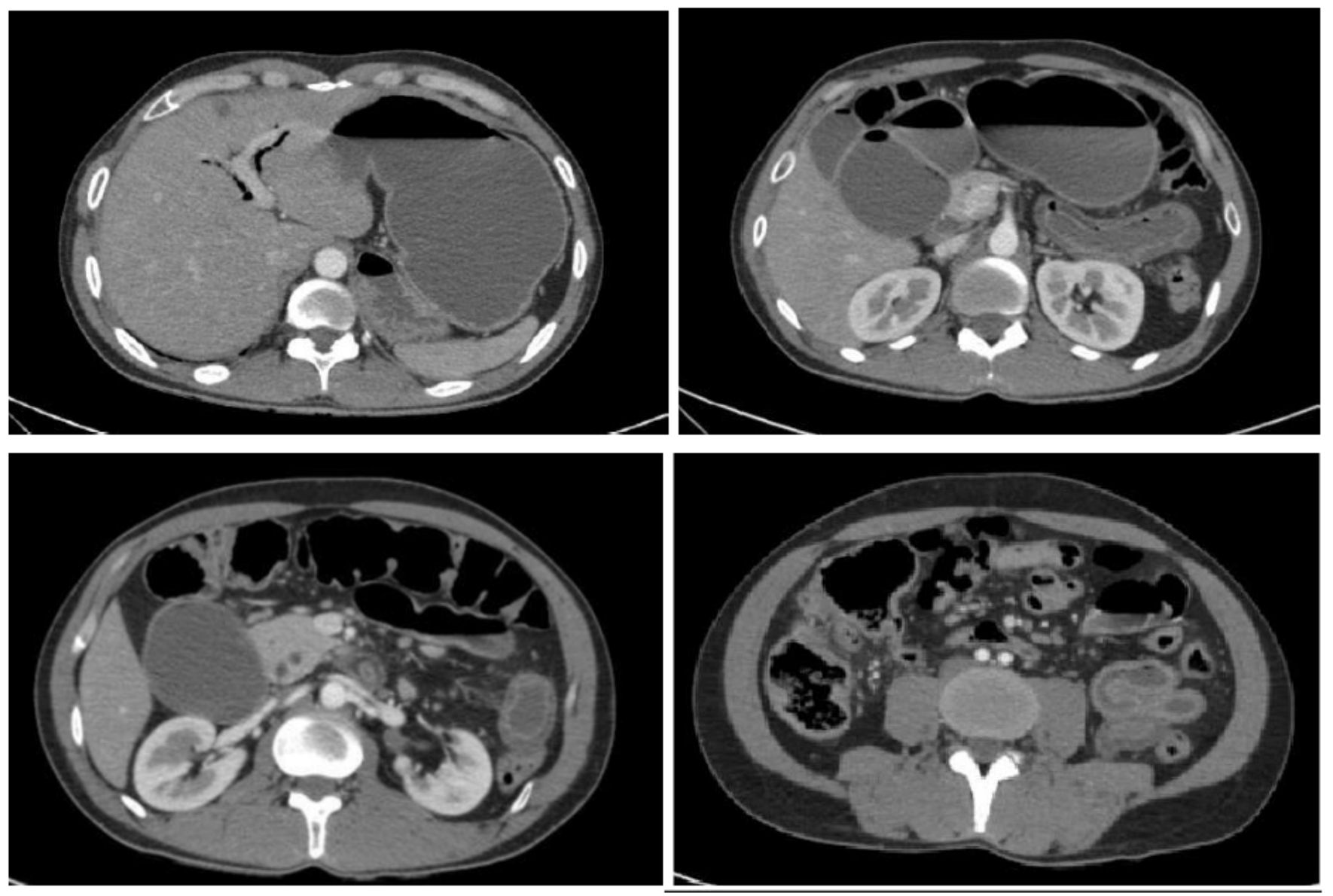

Figura 2 - TC de abdome: dilatação de vias biliares extra-hepáticas e com focos gasosos de permeio, distensão líquida à montante nas primeiras porções duodenais e câmara gástrica. Espessamento parietal de terceira porção do duodeno estendendo-se até o jejuno, medindo até $0,7 \mathrm{~cm}$ com realce de mucosa, com borramento dos contornos junto ao duodeno.

O anatomopatológico evidenciou infiltrado linfo-plasmocitário associado a numerosas formas adultas e larvas rabditiformes de Strongyloides stercoralis, localizadas em criptas. Teste rápido para HIV não reagente e anti-HTLV-1 reagente.

Mantido albendazol por 5 dias e iniciada a associação com tiabendazol, porém paciente apresentou cefaleia e hiporexia após início do medicamento. Optou-se por modificar para esquema com ivermectina $200 \mathrm{mcg} / \mathrm{kg} / \mathrm{dia}$ na qual foi realizado por 3 dias, sendo programado novos ciclos, 
2. Vadlamudi RS, Chi DS, Krishnaswamy G. Intestinal strongyloidiasis and hyperinfection syndrome. Clin Mol Allergy. 2006:4:8.

Available from: https://pubmed.ncbi.nlm.nih. gov/16734908/.

6. Gill GV, Welch E, Bailey JW, Bell DR, Beeching NJ. Chronic Strongyloides stercoralis infection in former British Far East prisoners of war. QJM. 2004;97(12):789-95.

7. Schulte C, Krebs B, Jelinek T, Nothdurft HD, von SF, Loscher T. Diagnostic significance of blood eosinophilia in returning travelers. Clin Infect Dis. 2002;34(3):407-11.

8. Roman-Sanchez P, Pastor-Guzman A, Moreno-Guillen S, Igual-Adell R, SunerGeneroso S, Tornero-Estebanez C. High prevalence of Strongyloides stercoralis among farm workers on the Mediterranean coast of Spain: analysis of the predictive factors of infection in developed countries. Am J Trop Med Hyg. 2003; 69(3):336-40. no total de quatro. Paciente apresentou demora para realizar parasitológico de fezes por quadro de constipação, sendo realizado após 2 semanas de tratamento, com resultado negativo. Evoluiu com melhora clínica, aceitando a dieta progressivamente até suspensão de dieta parenteral e alta hospitalar.

\section{DISCUSSÃO}

A estrongiloidíase crônica frequentemente cursa de forma assintomática, porém a forma grave apresenta alta taxa de mortalidade. Devido a relação com imunossupressão é importante investigar nos pacientes com quadros gastrointestinais ou pulmonares graves a possibilidade de parasitose intestinal.

É necessário ressaltar a importância do uso de medicamentos antiparasitários antes de corticoterapia imunossupressora devido a possibilidade de estrongiloidíase e risco de hiperinfecção, além da investigação de doenças infecciosas em pacientes com quadros graves em áreas tropicais e com saneamento básico precário. A associação entre HTLV-1 e estrongiloidíase tem sido demonstrada em regiões onde ambos os agentes são endêmicos.

O achado laboratorial mais importante observado em pacientes com estrongiloidíase é a eosinofilia, que mostra $93,5 \%$ de sensibilidade, com especificidade de $93,1 \%$ em populações de alto risco ${ }^{6,8}$.

Mas também é mostrado que a contagem de eosinófilos, se usada sozinha, não é suficientemente sensível para rastrear a doença, especialmente em pacientes com infecção crônica que podem ter contagens baixas ou normais de eosinófilos em alguns casos de síndrome de hiperinfecção e estrongiloidíase disseminada ${ }^{2,6,7}$.

Apesar do paciente apresentar fatores de risco pessoal como alcoolismo, condições precárias de saneamento e história familiar de câncer, é importante ressaltar a investigação de outras causas de imunossupressão, como infecções por HIV e HTLV que podem ser facilmente pesquisadas, auxiliarem no esclarecimento do caso e definir seguimento adequado. 


\section{REFERÊNCIAS}

1.Melo MCB,KlemVGQ, Mota JAC,Penna FJ.Parasitoses Intestinais. Rev Med Minas Gerais.2004;14(Suppl 1):3-12.

2. Vadlamudi RS, Chi DS, Krishnaswamy G. Intestinal strongyloidiasis and hyperinfection syndrome. Clin Mol Allergy. 2006:4:8. Available from: https://pubmed.ncbi.nlm.nih.gov/16734908/.

3. Brasil. Ministério da Saúde. Secretaria de Vigilância em Saúde. Departamento de Vigilância Epidemiológica. Doenças infecciosas e parasitárias: guia de bolso. 8th ed. Brasília: Distrito Federal; 2010. p.176-178. Available from: http://bvsms.saude.gov.br/bvs/publicacoes/doencas_infecciosas_ parasitaria_guia_bolso.pdf

4. Luna OB, Grasselli R, Ananias M, Pinto TS, Bozza FA, Soares M et al. Estrongiloidíase disseminada: diagnóstico e tratamento. Rev Bras Ter Intensiva. 2007;19(4):463-468. Available from: https://www.scielo.br/scielo.php?script=sci_arttext\&pid=S0103-507X2007000400010\&lng=pt\&nrm=iso \& tlng=pt.

5. Porto MAF, Muniz A, Oliveira Júnior J, Carvalho EM. Implicações clínicas e imunológicas da associação entre o HTLV-1 e a estrongiloidíase. Rev Soc Bras Med Trop. 2002;35(6):641649. Available from: https://www.scielo.br/scielo.php?script=sci_arttext\&pid=S0037$86822002000600016 \&$ lng=pt\&tlng=pt.

6. Gill GV, Welch E, Bailey JW, Bell DR, Beeching NJ. Chronic Strongyloides stercoralis infection in former British Far East prisoners of war. QJM. 2004;97(12):789-95.

7. Schulte C, Krebs B, Jelinek T, Nothdurft HD, von SF, Loscher T. Diagnostic significance of blood eosinophilia in returning travelers. Clin Infect Dis. 2002;34(3):407-11.

8. Roman-Sanchez P, Pastor-Guzman A, Moreno-Guillen S, Igual-Adell R, Suner- Generoso S, Tornero-Estebanez C. High prevalence of Strongyloides stercoralis among farm workers on the Mediterranean coast of Spain: analysis of the predictive factors of infection in developed countries. Am J Trop Med Hyg. 2003; 69(3):336-40. 